\title{
Mycobacterium chelonae bacteremia in a patient taking infliximab and prednisone
}

\author{
Caitlin McCallum MD, Bijai Johnson MD
}

Competing interests: None declared.

This article has been peer reviewed.

The authors have obtained patient consent.

Affiliations: Section of General Internal Medicine, Department of Internal Medicine, Faculty of Medicine, University of Manitoba, Winnipeg, Man.

\section{Acknowledgement:} The authors acknowledge Dr. Heather Adam from the National Microbiology Laboratory.

Correspondence to: Caitlin McCallum, cmccallum@nosm.ca

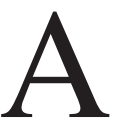
68-year-old man with ulcerative colitis was admitted for an elective colectomy and ileostomy after failed medical management. At the time of admission, he was otherwise well. He had been taking infliximab for 12 years. His prednisone dose was increased from 10 to $50 \mathrm{mg} / \mathrm{d}$ on admission. While awaiting surgery three days later, he developed fever, hypotension and a productive cough. He was given piperacillin-tazobactam and vancomycin for presumed pneumonia, despite a normal chest radiograph. On day 7 , his blood cultures were reported as positive for acid-fast bacilli, and his treatment was broadened to include rifampin, ethambutol, pyrazinamide, isoniazid and azithromycin to cover tuberculosis and Mycobacterium aviumintracellulare. On day 10, he developed multiple cutaneous nodules on his forearms that were $2-3 \mathrm{~cm}$ in diameter, well-demarcated

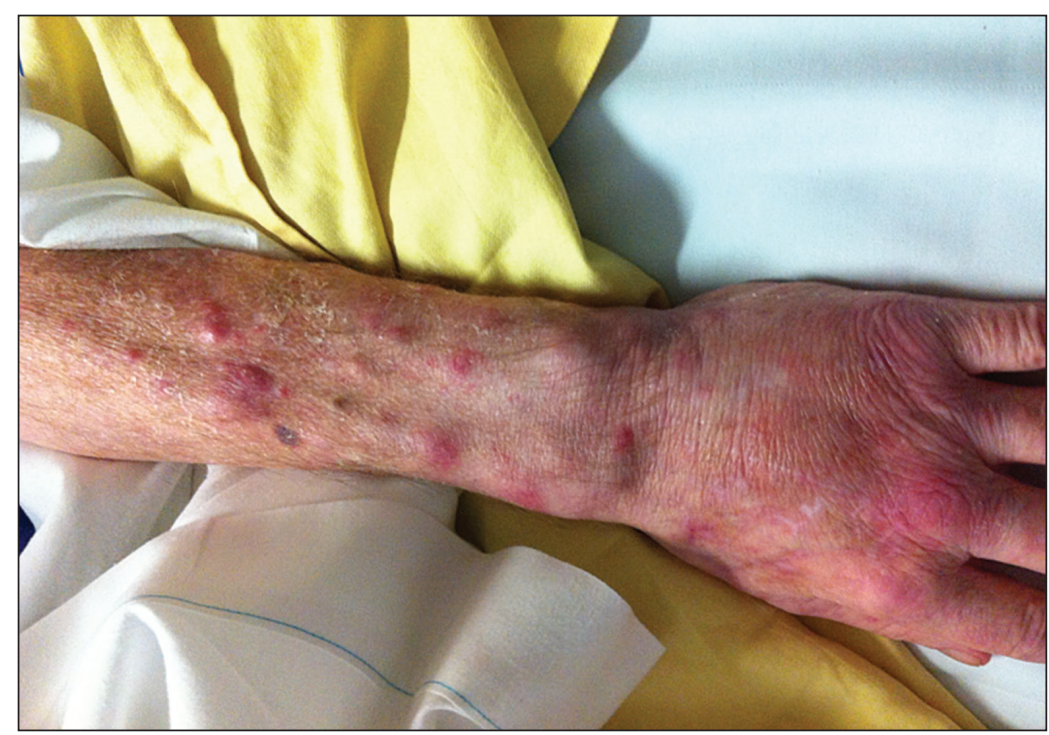

Figure 1: Left forearm of a 68-year-old man with cutaneous nodules associated with Mycobacterium chelonae infection. The patient was taking infliximab and prednisone for ulcerative colitis. and erythematous (Figure 1). No other source of infection was identified.

A sample of the mycobacteria isolated from the blood cultures was submitted to the National Microbiology Laboratory in Winnipeg and was identified as Mycobacterium chelonae subsp. niacinogenes. Sensitivities are displayed in Appendix 1, available at www.cmaj.ca/lookup/ suppl/doi:10.1503/cmaj.150978/-/DC1. The patient's treatment was consolidated to clarithromycin owing to rapid improvement in his symptoms. His elective surgical procedure was rescheduled.

Nontuberculous mycobacteria infections are increasing in prevalence and incidence, ${ }^{1}$ likely owing to advances in identification of nontuberculous mycobacteria and increasing use of immunosuppressive agents. ${ }^{2}$

Mycobacterium chelonae is a rapidly growing nontuberculous mycobacteria. Most infections involving $M$. chelonae are limited to cutaneous lesions and have been reported with multiple surgical interventions, including cosmetic procedures, otolaryngologic procedures and cardiovascular surgery. ${ }^{3}$ Disseminated infection has been reported in patients using contaminated hemodialysis machines and in immunosuppressed patients with infected vascular devices. ${ }^{4}$ In our patient, the presumed inoculation occurred with a peripheral intravenous line.

\section{References}

1. Wentworth AB, Drage L, Wengenack N, et al. Increased incidence of cutaneous nontuberculous mycobacterial infection, 1980 to 2009: a population-based study. Mayo Clin Proc 2013; 88:38-45.

2. Griffith DE, Aksamit T, Brown-Elliott B, et al. An official ATS/IDSA statement: diagnosis, treatment, and prevention of nontuberculous mycobacterial diseases [published erratum in Am J Respir Crit Care Med 2007;175:744-5]. Am J Respir Crit Care Med 2007; 175:367-416.

3. Falkinham JO. Epidemiology of infection of nontuberculous mycobacteria. Clin Microbiol Rev 1996;9:117-215.

4. Hay RJ. Mycobacterium chelonae - a growing problem in soft tissue infection. Curr Opin Infect Dis 2009;22:99-101. 